\title{
Improving the evaluation of COPD exacerbation treatment effects by accounting for early treatment discontinuations: a post-hoc analysis of randomized clinical trials
}

\author{
Agnieszka Król ${ }^{1 \dagger}$, Robert Palmér ${ }^{1 \dagger}$, Virginie Rondeau ${ }^{2}$, Stephen Rennard ${ }^{3,4}$, UIf G. Eriksson ${ }^{1}$ and
} Alexandra Jauhiainen ${ }^{5^{*}}$ (1)

\begin{abstract}
Background: Chronic obstructive pulmonary disease (COPD) clinical trials aimed at evaluating treatment effects on exacerbations often suffer from early discontinuations of randomized treatment. Treatment discontinuations imply a loss of information and should ideally be considered in the statistical analysis of trial results, particularly if the discontinuations are related to the disease or treatment itself. Here, we explore this issue by investigating (1) whether there exists an association between the risks of exacerbation and treatment discontinuation in COPD clinical trials and (2) whether disregarding this association can cause bias in exacerbation treatment effect estimates. We focus on the hypothetical estimand, i.e. the treatment effect that would have been observed had all subjects completed the trial as planned.

Methods: The association between exacerbation and discontinuation risks was analysed by applying a joint frailty (random effect) model - allowing for the simultaneous analysis of multiple types of correlated events - to data from five Phase III-IV COPD clinical trials. Specifically, the impact of the association on exacerbation treatment effect estimates was assessed by comparing the treatment hazard ratios of the joint frailty model to the rate/hazard ratios of two related statistical models (the negative binomial and shared frailty models), which both assume discontinuations to be unrelated to the trial outcome. The models were also compared using simulated data.

(Continued on next page)
\end{abstract}

\footnotetext{
* Correspondence: alexandra.jauhiainen@astrazeneca.com

${ }^{+}$Agnieszka Król and Robert Palmér contributed equally to this work.

${ }^{5}$ BioPharma Early Biometrics and Statistical Innovation, Data Science \& Al,

BioPharmaceuticals R\&D, AstraZeneca, Pepparedsleden 1, SE-431 83 Mölndal,

Sweden

Full list of author information is available at the end of the article
}

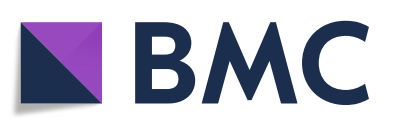

(- The Author(s). 2020 Open Access This article is licensed under a Creative Commons Attribution 4.0 International License, which permits use, sharing, adaptation, distribution and reproduction in any medium or format, as long as you give appropriate credit to the original author(s) and the source, provide a link to the Creative Commons licence, and indicate if changes were made. The images or other third party material in this article are included in the article's Creative Commons licence, unless indicated otherwise in a credit line to the material. If material is not included in the article's Creative Commons licence and your intended use is not permitted by statutory regulation or exceeds the permitted use, you will need to obtain permission directly from the copyright holder. To view a copy of this licence, visit http://creativecommons.org/licenses/by/4.0/. The Creative Commons Public Domain Dedication waiver (http://creativecommons.org/publicdomain/zero/1.0/) applies to the data made available in this article, unless otherwise stated in a credit line to the data. 
(Continued from previous page)

Results: A statistically significant $(p<0.0001)$, positive association between exacerbation and discontinuation risks was found in all trials. Importantly, simulations confirmed that - with such an association - models disregarding the association risk producing biased results (> 5 percentage point difference in hazard/rate ratio). For some treatment comparisons in the clinical trials, the difference in treatment effect estimates between the joint frailty and the other models was as high as 10-15 percentage points. The difference was affected by the strength of the exacerbationdiscontinuation association, the population heterogeneity in exacerbation risk, and the difference in discontinuation rates between treatment arms.

Conclusions: We have identified an association between the risks of exacerbation and treatment discontinuation in five COPD clinical trials. We recommend using the joint frailty model to account for this association when estimating exacerbation treatment effects, particularly when targeting the hypothetical estimand.

Keywords: COPD, Early treatment discontinuations, Dropouts, Exacerbations, Joint frailty model, Recurrent events, Survival analysis

\section{Background}

In late-phase, randomized chronic obstructive pulmonary disease (COPD) clinical trials, a significant number of patients often discontinue their randomized treatment before the planned end of the trial $[1,2]$. Many of these early treatment discontinuations can be associated to e.g. disease worsening or adverse events and thus be considered as informative censoring events. As such, they may influence the estimation of treatment effects and lead to biased trial outcomes if not handled properly [3-6].

Herein, we investigate the impact of early treatment discontinuations on COPD exacerbation treatment effect estimates. COPD exacerbations are often used as a key endpoint in late-phase COPD clinical trials. However, the appropriate statistical approach used to analyse them is a topic of scientific debate [7-11]. Typically, exacerbations are analysed either as a rate endpoint using negative binomial regression or as a time-to-first event endpoint using the Cox proportional hazards model [12-14], but both these approaches have limitations.

Particularly in longer clinical trials ( $\geq 6$ months), where patients may experience multiple exacerbations, information may be lost if only the time-to-first exacerbation is used as in a standard Cox analysis. Moreover, the Cox analysis typically neglects patient heterogeneity in exacerbation risk and assumes that, given the same set of covariates (e.g. treatment and potential prognostic factors), the risk for each patient is the same. The negative binomial model, on the other hand, can handle both multiple events per subject and unobserved risk heterogeneity but instead assumes constant risk over time and ignores the timing and ordering of events.

One way to alleviate some of the assumptions of standard methods would be to use the shared frailty model [15]. The shared frailty model is based on the AndersenGill (AG) counting process model, a generalization of the Cox model for the analysis of recurrent events [16]. Extending the AG model to the shared frailty model is done by introducing a random effect called frailty. Not unlike its medical interpretation, the term frailty is used to indicate that some individuals may have a higher risk of an event (in our case exacerbations) than others. That is, like the random effect in the negative binomial model, the frailty describes risk heterogeneity and allow for the analysis of correlated recurrent events within individuals in a flexible way. Importantly, though, neither the shared frailty nor standard models address the issue of informative censoring. Instead, the assumption that discontinuations are unrelated to the trial outcome, i.e. that exacerbation data are missing at random (MAR), is often used with these models when the aim is to estimate the treatment effect as if all subjects would have completed the trial as planned, i.e. when targeting the de jure or hypothetical estimand $[10,17,18]$.

Keene et al. [10, 19] have previously suggested using various imputation methods to study the sensitivity of exacerbation treatment effect estimates to the MAR assumption, focusing on the negative binomial model and treatment policy estimand [17]. Here, however, we consider a novel approach to particularly address the hypothetical estimand. This approach involves the use of a joint frailty model $[20,21]$ for the simultaneous analysis of recurrent episodes of moderate/severe exacerbations and early treatment discontinuations as two semi-competing, possibly correlated, types of events. Specifically, we hypothesize that there exists an association between a patient's risk of exacerbation and risk early treatment discontinuation and that disregarding this association in the statistical analysis may lead to biased treatment effect estimates. To investigate this hypothesis, we perform a post-hoc analysis and compare the treatment hazard ratios of the joint frailty model to the hazard/rate ratios of the shared frailty and negative binomial models using both simulated data and patient-level data from five Phase III-IV clinical trials in moderate-to-severe 
COPD patients. We also use the clinical trial data to identify and adjust the joint frailty model with key prognostic factors of exacerbation and discontinuation risks.

\section{Methods}

\section{Clinical trial datasets}

Patient-level data from five Phase III-IV, randomized, double-blind, parallel-group, multicentre trials including a total of 7698 patients with moderate-to-severe COPD served as the basis for this post-hoc analysis (Table 1). The trials were selected to be long and large enough to provide reasonable numbers of early treatment discontinuations and exacerbations to enable appropriate analysis of the association between them (moderate/severe exacerbations were included as either a primary or secondary endpoint in all trials). From the perspective of this analysis, there were no important differences in the design of the Phase III and Phase IV trials.

Trials A [22], B [23], and C [24] were of 6-12 months duration and evaluated the efficacy of combinations of budesonide (inhaled corticosteroid, ICS) and formoterol (inhaled long-acting beta agonist, LABA) in moderate-tosevere COPD patients. Trials D [13] and E [14] were both of 12 months duration and evaluated the efficacy of roflumilast (oral phosphodiesterase 4 inhibitor) on top of standard of care (SOC) treatment with ICS and LABA, plus/ minus a long-acting muscarinic receptor antagonist (LAMA), in patients with severe COPD. All trials were conducted in accordance with the Declaration of Helsinki, the International Conference on Harmonisation Guidelines for
Good Clinical Practice, and applicable regulatory requirements. For further details on the clinical trials, see Additional file 1 and the original trial publications.

Before the analysis, all informed consent forms were reviewed for data re-use in accordance with AstraZeneca data sharing rules. Patients from countries where ethics committees did not approve data re-use, as well as patients who had withdrawn consent, were excluded. Consequently, only a subset of the original trial data were part of this analysis and any direct comparison with the original trial results should be made with care. We refer to the subsets of data as Datasets A-E (see Table 1 for a comparison of the number of patients included in the original trials and in this analysis).

\section{Definition of exacerbations and early discontinuations}

A COPD exacerbation was defined as an episode of worsening of respiratory symptoms requiring additional treatment with oral corticosteroids, antibiotics, and/or hospitalization (i.e. moderate/severe exacerbations). The start and end time of an exacerbation was defined as the number of days from randomization to first initiation and last cessation of additional treatments, respectively. Patients were considered not at risk during an exacerbation episode.

Early treatment discontinuations were defined as any discontinuation of randomized treatment before the planned end of treatment day. The time of discontinuation was defined as the day of the last dose of the randomized treatment. End-of-trial completion was treated as a non-informative right-censoring event. Reasons for

Table 1 Summary of clinical trial datasets

\begin{tabular}{|c|c|c|c|c|c|}
\hline Dataset & $\mathrm{N}$ (original) & $\mathrm{N}$ (analysis) & Duration (months) & Treatment groups* & Endpoints \\
\hline $\mathrm{A}$ & 1964 & 1746 & 12 & 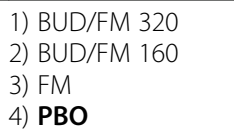 & $\begin{array}{l}\text { Primary: pre- and } \\
\text { post-dose FEV } \\
\text { Secondary: number of exacerbations }\end{array}$ \\
\hline B & 1219 & 1072 & 12 & $\begin{array}{l}\text { 1) BUD/FM } 320 \\
\text { 2) BUD/FM } 160 \\
\text { 3) FM }\end{array}$ & Primary: number of exacerbations \\
\hline C & 1704 & 1613 & 6 & $\begin{array}{l}\text { 1) BUD/FM } 320 \\
\text { 2) BUD/FM } 160 \\
\text { 3) BUD } 320+F M \\
\text { 4) BUD } 320 \\
\text { 5) FM } \\
\text { 6) } \mathrm{PBO}\end{array}$ & $\begin{array}{l}\text { Primary: pre- and post-dose } \mathrm{FEV}_{1} \\
\text { Secondary: number of exacerbations }\end{array}$ \\
\hline D & 1945 & 1218 & 12 & $\begin{array}{l}\text { 1) } S O C+R F L \\
\text { 2) SOC }\end{array}$ & $\begin{array}{l}\text { Primary: rate of } \\
\text { exacerbations }\end{array}$ \\
\hline E & 2354 & 2049 & 12 & $\begin{array}{l}\text { 1) } S O C+R F L \\
\text { 2) } S O C\end{array}$ & $\begin{array}{l}\text { Primary: rate of } \\
\text { exacerbations }\end{array}$ \\
\hline
\end{tabular}

BUD budesonide, $F E V$ forced expiratory volume in $1 \mathrm{~s}, F M$ formoterol, PBO placebo, RFL roflumilast, SOC standard of care, * - Treatment groups: BUD/FM 320 BUD/FM pMDI (pressurized metered-dose inhaler) $160 / 4.5 \mu \mathrm{g} \times 2$ inhalations bid (twice daily) $(320 / 9 \mu \mathrm{g}), \mathrm{BUD} / \mathrm{FM} 160-\mathrm{BUD} / \mathrm{FM} \mathrm{pMDI} 80 / 4.5 \mu \mathrm{g} \times 2$ inhalations bid $(160 / 9 \mu \mathrm{g}), \mathrm{FM}-\mathrm{FM}$ DPI (dry powder inhaler) $4.5 \mathrm{mcg} \times 2$ inhalations bid $(9 \mu \mathrm{g})$, BUD $320-$ BUD pMDI $160 \mu \mathrm{g} \times 2$ inhalations bid $(320 \mu \mathrm{g}), \mathrm{BUD} 320+\mathrm{FM}-\mathrm{BUD}$ pMDI $160 \mu \mathrm{g} \times 2$ inhalations bid $(320 \mu \mathrm{g})$ and FM DPI $4.5 \mu \mathrm{g} \times 2$ inhalations bid $(9 \mu \mathrm{g})$, PBO - use of SABA (short-acting beta agonists) only, SOC - standard of care with inhaled corticosteroid and long-acting beta agonist $+/$ - long-acting muscarinic receptor agonist, SOC + RFL - standard of care and RFL $500 \mu g$ once daily. The reference treatment in each dataset is marked in bold 
early treatment discontinuations (to the extent known) in the different studies are summarised in Additional file

\section{1: Table S1.}

\section{Statistical analysis}

Statistical analyses were carried out using $\mathrm{R}$ version 3.4.3 [25] (packages listed in Additional file 1).

Recurrent episodes of moderate/severe exacerbations and early treatment discontinuations were simultaneously analysed using a joint frailty model [26], at first with treatment as the only covariate (for both exacerbations and discontinuations). The model (Fig. 1) consists of two sub-models - an Andersen-Gill model for recurrent episodes of exacerbations and a time-to-first event proportional hazards model for early discontinuations linked using a gamma-distributed random effect (frailty). The frailty describes the between-patient variability in exacerbation (and discontinuation) risk and acts proportionally on the baseline hazards, which are approximated with splines allowing for time-varying risk. A frailty variance of zero would indicate that all subjects (with the same set of covariates) have the same risk of experiencing an event, while an increasing frailty variance implies an increasing between-patient difference in risk, as well as an increasing difference in the number of events per subject. In the discontinuation hazard function, the frailty is scaled by an exponent $(\alpha)$ describing the strength of the association between discontinuation and exacerbation risks. The value of $\alpha$ is valid if the estimated variance of the frailty is significantly greater than zero, with the interpretation that for $\alpha>0$ and $\alpha<0$ the risks are positively and negatively associated, respectively. If $\alpha=0$, the risks are considered unrelated, and the joint frailty model reduces to the shared frailty model (for the exacerbation part, Fig. 1). Wald-tests were used to test if $\alpha \neq 0$ and frailty variance $>0$. For more details, see Additional file 1 .

Firstly, the impact of disregarding an association between exacerbation and treatment discontinuation risks was investigated using simulated data from the joint frailty model. Simulating data allowed us to investigate in a controlled way - what properties of exacerbation and discontinuation data may cause biased treatment effect estimates. The simulated data included two treatment groups (control and active) with overall exacerbation and discontinuation rates, as well as number of patients, chosen to be similar to what we observed in Datasets A-E. Several scenarios were evaluated: 1) different strengths of the risk association, 2) different levels of frailty variance, and 3) different discontinuation rates in the two treatment groups.

After simulating data (1000 datasets per scenario), the effect of disregarding the association in the statistical analysis was evaluated by fitting a shared frailty model to the data and comparing the estimated exacerbation hazard ratios to the "true" hazard ratio used in the simulations. The joint frailty model itself was also fitted to the data for comparison, to make sure it produced unbiased estimates of the hazard ratio. See Additional file 1 for further information on how the simulation study was done.

Secondly, the joint frailty model was applied to the clinical trial data (Datasets A-E) and exacerbation hazard ratio estimates of the model were compared to the hazard/rate ratios of both the shared frailty and negative binomial models. Hazard/rate ratios were calculated using the original comparator arm in each trial as reference, i.e. placebo in Datasets A and C, formoterol in Dataset

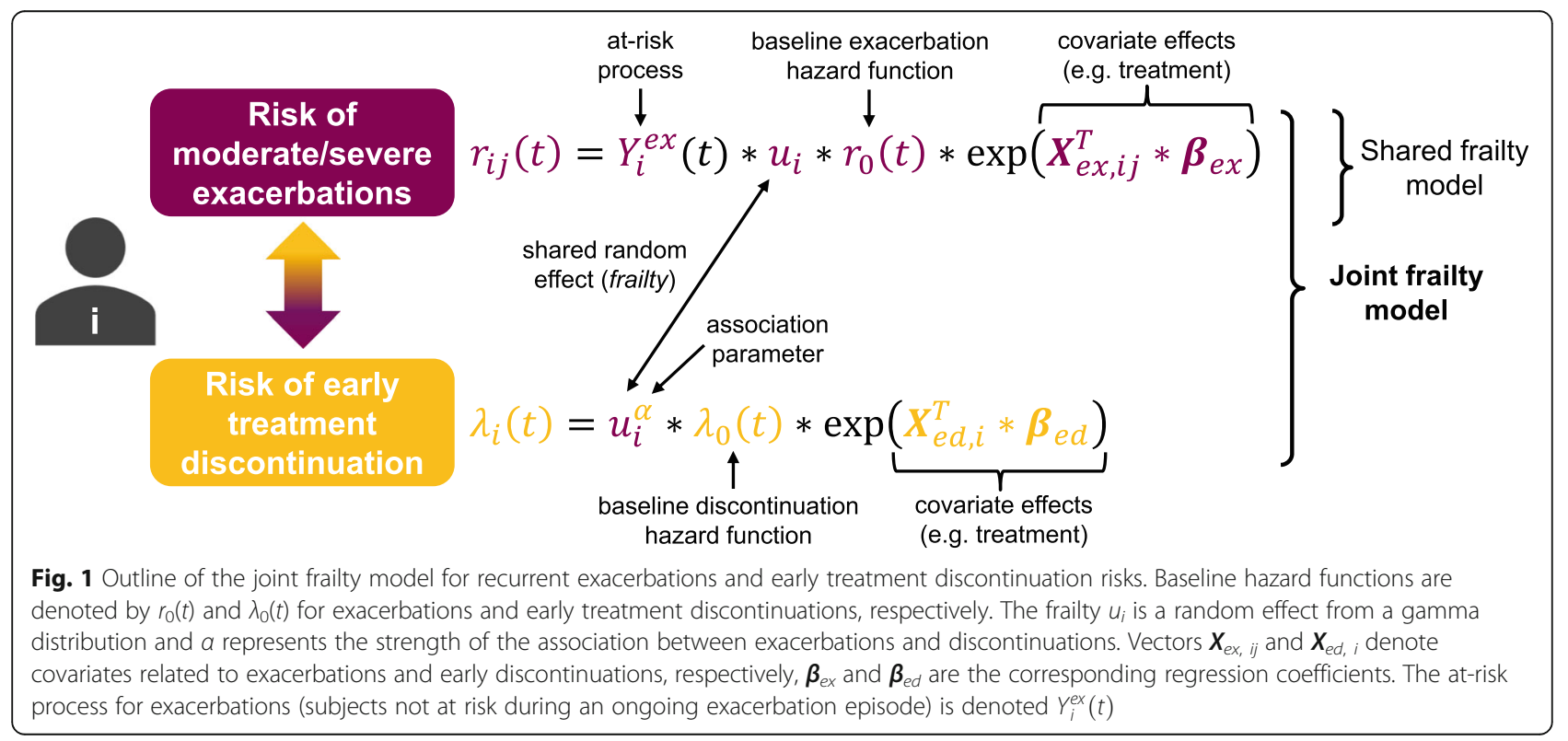


B, and SOC in Datasets D and E (Table 1). We decided not to include a comparison with a standard time-tofirst-event Cox analysis, since the focus of this work is on the impact of treatment discontinuations and not on the comparison of time-to-first-event and recurrentevent analyses.

Finally, the joint frailty model was further evaluated by adjusting for prognostic factors of exacerbation and discontinuation risks and evaluating their impact on the frailty variance and risk association. Prognostic factors were selected based on an extensive covariate search using pooled data from Datasets A-C and D-E (see Additional file 1 for details). First, LASSO Cox regression [27] was performed separately on exacerbation and discontinuation data. The most frequently selected covariates were then complemented with other common prognostic factors, and stepwise backwards selection using the joint frailty model was performed. The tested covariates included patient demographic and disease history data, as well as baseline measures of clinical lab variables, spirometry variables, and patient reported outcomes (PROs), such as diary recordings, St. George's Questionnaire (SGRQ), and the COPD Assessment Test $^{\text {mi }}$ (CAT). Seasonality - a known predictor of exacerbation risk [28] - was also added to the model as a time-varying covariate.

\section{Results}

\section{Clinical data characteristics}

Exacerbation and discontinuation data for Datasets A-E are summarised in Table 2. The percentage of patients with at least one moderate/severe exacerbation and the overall number of moderate/severe exacerbations per patient time on treatment varied between 28 and 55\% and
0.816-1.293 events/year, respectively. Early treatment discontinuation frequencies were between 15 and 23\%.

Table 2 also shows the discontinuation frequencies in the original trials. As can be seen, original frequencies are higher than in our datasets, indicating that many of the patients who had to be excluded in this analysis, due to data re-use rules, were patients who also had discontinued treatment.

Kaplan-Meier curves of early treatment discontinuations for each dataset and treatment arm are summarised in Fig. 2 together with corresponding hazard ratios. In Datasets A-C, treatments considered more effective in terms of disease control (combinations of budesonide and formoterol) were associated with a reduced risk of early treatment discontinuation compared to less effective treatments arms (formoterol or placebo), with discontinuation hazard ratios between 0.55-0.99. In Datasets D-E, on the other hand, higher rates of early treatment discontinuations were seen in the roflumilast arms compared to SOC treatment alone (discontinuation hazard ratios 1.48 and 1.69).

\section{Analysis of simulated data}

The parameters used for simulating exacerbation and discontinuation data are described in Fig. 3. The figure also illustrates the difference (bias) between the "true" and estimated exacerbation treatment effect of a shared frailty model when applied to the simulated data. The shared frailty model was found to underestimate the treatment effect with a difference in hazard ratio of $\sim 7$ percentage points $(0.67$ compared to $0.6)$ in cases of high frailty variance, a strong risk association, and a protective treatment effect on treatment discontinuations (discontinuation hazard ratio $=$

Table 2 Summary data of analysis datasets with respect to recurrent exacerbations and early discontinuations

\begin{tabular}{|c|c|c|c|c|c|}
\hline & Dataset A & Dataset B & Dataset C & Dataset D & Dataset E \\
\hline Number of patients available for analysis & 1746 & 1072 & 1613 & 1218 & 2049 \\
\hline Number of mod/sev exacerbations & 1186 & 949 & 655 & 1350 & 2179 \\
\hline \multicolumn{6}{|l|}{ Number of patients with $\mathrm{R}$ number of mod/sev exacerbations } \\
\hline$R=0$ & 1086 & 537 & 1170 & 604 & 920 \\
\hline$R=1$ & 376 & 286 & 301 & 265 & 554 \\
\hline$R \geq 2$ & 284 & 249 & 142 & 349 & 575 \\
\hline $\begin{array}{l}\text { Percentage of patients } \\
\text { with } \geq 1 \mathrm{mod} / \mathrm{sev} \text { exacerbation }\end{array}$ & $37.8 \%$ & $49.9 \%$ & $27.5 \%$ & $50.4 \%$ & $55.1 \%$ \\
\hline Max. number of mod/sev exacerbations & 12 & 10 & 6 & 11 & 11 \\
\hline Mean number of mod/sev exacerbations per patient years at risk & 0.816 & 1.051 & 0.917 & 1.284 & 1.293 \\
\hline Number of early treatment discontinuations & 392 & 221 & 235 & 180 & 421 \\
\hline Percentage of early treatment discontinuations & $22.5 \%$ & $20.6 \%$ & $14.6 \%$ & $14.8 \%$ & $20.5 \%$ \\
\hline Percentage of early treatment discontinuations in original data & $31 \%$ & $30 \%$ & $19 \%$ & $24 \%$ & $25 \%$ \\
\hline
\end{tabular}


Dataset A

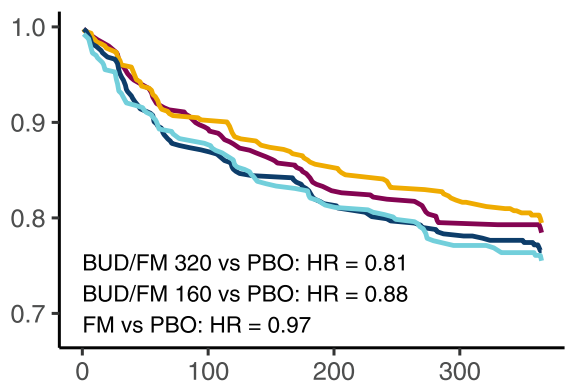

Dataset C

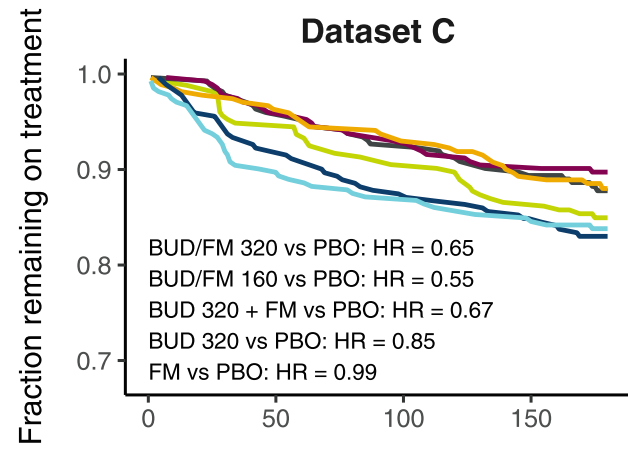

Dataset E

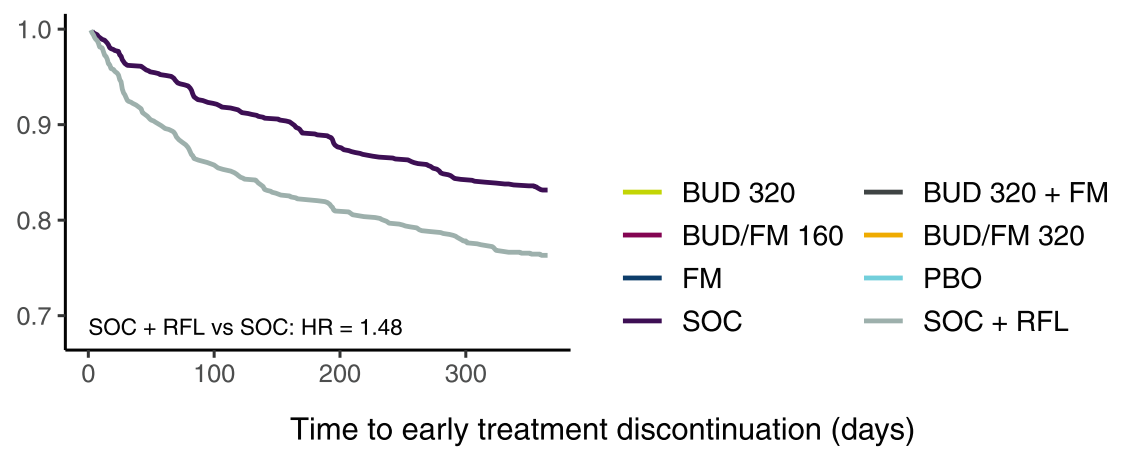

Dataset B

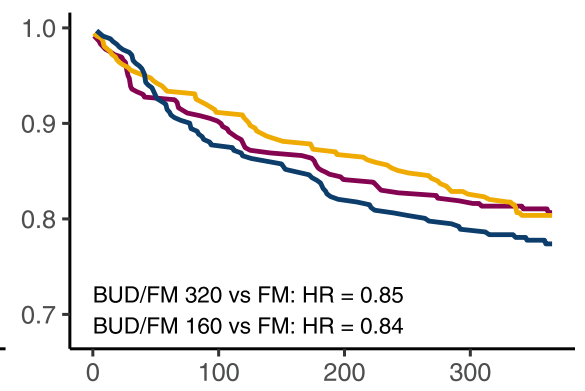

Dataset D

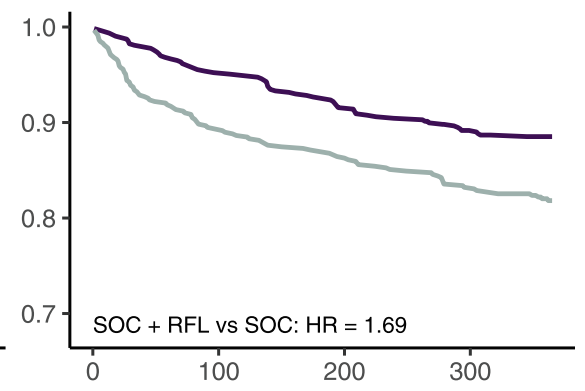

Fig. 2 Kaplan-Meier curves for time to early treatment discontinuation for each treatment arm in datasets A-E. The hazard ratio (HR, from a proportional hazards model) is given for the relevant comparisons in each dataset. BUD - budesonide, FM - formoterol, PBO - placebo, RFL roflumilast, SOC - standard of care

0.5). Conversely, the treatment effect was overestimated if active treatment caused more discontinuations than the control arm (discontinuation hazard ratio $=2$ ). A tendency of overestimation was also seen when there was no difference in discontinuation rate between the two groups. Overall, the bias got smaller with smaller frailty variance and weaker risk association.

Results using other exacerbation and treatment discontinuations rates are found in Additional file 1: Figure S1, highlighting that the overall treatment discontinuation rate in a trial is also an important factor affecting the bias (increased rate leads to increased bias). Using the joint frailty model to estimate the hazard ratio resulted in no bias (Additional file 1: Figure S2).

\section{Analysis of clinical trial data}

Statistically significant frailty variances and exacerbationdiscontinuation associations was found in all clinical trial datasets $(p<0.0001$ for both parameters in all datasets, Table 3). The parameter estimates for the frailty variance and association varied between $0.74-2.17$ and $0.83-1.84$, respectively. The lowest association was seen in the two roflumilast datasets (Datasets D-E).

A comparison of the estimated exacerbation hazard ratios of the joint frailty model with the hazard/rate ratios of the shared frailty and negative binomial models is shown in Table 4. In summary, the joint frailty model consistently estimated larger treatment effects than the other models for treatments with an early protective effect on treatment discontinuations, and smaller treatment effects in the reverse situation. The shared frailty 




Fig. 3 Hazard ratios for exacerbations estimated using a shared frailty model fitted to simulated data. Data were generated using a joint model with a gamma-distributed frailty with different levels of frailty variance, different association between exacerbation and discontinuation risks, and different treatment discontinuation rates between the two treatment arms. The mean (overall) rate of exacerbations was set to 0.9 per year, the overall percentage of discontinuations to $25 \%$, and the true hazard ratio for the risk of exacerbations to 0.6 in all scenarios. The $y$-axis shows the estimated median hazard ratio across 1000 simulations

and negative binomial models produced similar estimates across all datasets and treatment arms.

For Datasets A-C, the difference in hazard/rate ratios between the joint frailty and the other models ranged between 0 and 15 percentage points, with the most pronounced difference seen in Dataset C. Of note is that, in the one case where the joint frailty model did not estimate a larger treatment effect (formoterol/budesonide $160 \mu \mathrm{g}$, Dataset B), there is a higher number of very early treatment discontinuations in the treatment arm compared to the reference (formoterol only) arm (Fig. 2).

For Dataset D-E, the joint model estimated smaller treatment effects than the shared frailty and negative binomial models, with a difference in hazard/rate ratio of 4-6 percentage points. This is in line with the discontinuation patterns seen in these datasets (Fig. 2), with roflumilast-treated patient having an increased treatment discontinuation rate.

Table 3 Estimates of the association parameters for recurrent exacerbations and discontinuations using the joint frailty model

\begin{tabular}{|c|c|c|}
\hline & Frailty variance & Association (a) \\
\hline & \multicolumn{2}{|c|}{ Estimate $(\mathrm{SE})^{*}$} \\
\hline Dataset A & $1.84(0.13)$ & $0.99(0.11)$ \\
\hline Dataset B & $0.87(0.10)$ & $1.84(0.24)$ \\
\hline Dataset C & $2.17(0.20)$ & $1.28(0.23)$ \\
\hline Dataset D & $1.34(0.11)$ & $0.93(0.18)$ \\
\hline Dataset E & $0.74(0.06)$ & $0.83(0.16)$ \\
\hline
\end{tabular}

$S E$ standard error, * - all parameters significantly different from zero with $p$-value $<0.0001$
Additional parameter estimates, such as the frailty variance and dispersion in the shared and negative binomial models, and the discontinuation hazard ratios in joint models, can be found in Additional file 1: Tables S2-S3.

\section{Covariate search}

Three disease factors common to pooled Datasets A-C and D-E were found to be significantly associated with exacerbation risk in the covariate search: number of exacerbations in previous year, baseline $\mathrm{FEV}_{1}$, and baseline reliever medication use (Table 5). In addition, previous ICS and/or LABA use, smoking history, baseline breathlessness score, and baseline SGRQ score were identified as statistically significant covariates in pooled Dataset A$\mathrm{C}$, while baseline CAT score was identified as an influential covariate in pooled Dataset D-E. Geographic region was also found to be associated with exacerbation risk in both pooled datasets, with a higher risk of exacerbations in the US and Western Europe compared to other parts of the world. Adding seasonality as a time-varying covariate indicated that exacerbation risk varies significantly with season, with $a \sim 30 \%$ lower risk during spring and summer compared to autumn and winter.

For early treatment discontinuations, age, sex, geographic region, baseline $\mathrm{FEV}_{1}$, baseline SGRQ score, baseline CAT score, and baseline reliever medication use were - in addition to treatment - identified as statistically significant covariates in one or both pooled datasets (Table 5). Noteworthy is that, even after including the covariates for both exacerbations and discontinuations, 
Table 4 Estimated exacerbation treatment effects and 95\% confidence intervals for the different models in Datasets A-E

\begin{tabular}{|c|c|c|c|}
\hline & Joint frailty model & Shared frailty model & Negative binomial model \\
\hline & \multicolumn{3}{|c|}{ Exacerbation treatment ratio ( $95 \%$ confidence interval)* } \\
\hline \multicolumn{4}{|l|}{ Dataset A } \\
\hline BUD/FM 320 vs PBO & $0.59(0.45-0.77)$ & $0.63(0.49-0.81)$ & $0.63(0.49-0.80)$ \\
\hline BUD/FM 160 vs PBO & $0.58(0.44-0.75)$ & $0.60(0.46-0.77)$ & $0.60(0.47-0.77)$ \\
\hline FM vs PBO & $0.90(0.69-1.17)$ & $0.90(0.70-1.16)$ & $0.90(0.71-1.14)$ \\
\hline \multicolumn{4}{|l|}{ Dataset B } \\
\hline BUD/FM 320 vs FM & $0.67(0.54-0.82)$ & $0.68(0.55-0.83)$ & $0.68(0.55-0.83)$ \\
\hline BUD/FM 160 vs FM & $0.74(0.60-0.92)$ & $0.74(0.60-0.90)$ & $0.73(0.59-0.89)$ \\
\hline \multicolumn{4}{|l|}{ Dataset C } \\
\hline BUD/FM 320 vs PBO & $0.73(0.50-1.08)$ & $0.87(0.60-1.25)$ & $0.88(0.62-1.24)$ \\
\hline BUD/FM 160 vs PBO & $0.70(0.47-1.04)$ & $0.83(0.58-1.20)$ & $0.84(0.59-1.20)$ \\
\hline BUD $320+$ FM vs PBO & $0.57(0.38-0.85)$ & $0.67(0.46-0.98)$ & $0.68(0.47-0.98)$ \\
\hline BUD 320 vs PBO & $0.72(0.48-1.07)$ & $0.81(0.55-1.18)$ & $0.82(0.57-1.17)$ \\
\hline FM vs PBO & $1.09(0.74-1.61)$ & $1.15(0.80-1.66)$ & $1.16(0.82-1.63)$ \\
\hline \multicolumn{4}{|l|}{ Dataset D } \\
\hline SOC + RFL vs SOC & $0.82(0.68-0.98)$ & $0.76(0.64-0.90)$ & $0.76(0.64-0.90)$ \\
\hline \multicolumn{4}{|l|}{ Dataset E } \\
\hline $\mathrm{SOC}+\mathrm{RFL}$ vS SOC & $0.95(0.85-1.07)$ & $0.91(0.81-1.02)$ & $0.91(0.81-1.02)$ \\
\hline
\end{tabular}

the remaining unexplained risk heterogeneity (frailty variance) was still statistically significant and only about $30 \%$ lower than in the corresponding models without covariates (Table 5 vs. Additional file 1: Table S4). All tested and included covariates are summarised in Additional file 1: Tables S5-S6 and Figure S3. Exacerbation and early treatment discontinuation baseline hazard functions are illustrated in Additional file 1: Figure S4.

\section{Discussion}

Using a joint frailty model, we have identified a significant association between exacerbation and early treatment discontinuation risks in data from five Phase III-IV COPD clinical trials. Importantly, we show that ignoring this association in the statistical analysis of exacerbation treatment effects risks leading to substantially biased results, particularly when targeting the hypothetical estimand. Since biased statistical results may lead to incorrect conclusions regarding the effectiveness of treatments, we consider it important to thoroughly investigate this issue in future analyses of COPD clinical trials (e.g. by using the joint frailty model).

It is known that early treatment discontinuations can constitute an important source of bias in the estimates of treatment effects in clinical trials [4]. Patients with more severe disease, or patients experiencing lack of treatment effect or adverse events, may have an increased tendency to discontinue treatment [29, 30]. Interestingly, we found that regardless of the actual treatment effect on discontinuations, there is an underlying positive association between discontinuation and exacerbation risks in COPD trials. Specifically, we see that irrespectively of analysing combinations of budesonide and formoterol (shown to reduce treatment discontinuation rates compared to single treatment or placebo), or roflumilast (shown to increase treatment discontinuation rates, primarily due to gastrointestinal (GI) adverse events) [30], the association between risks - after adjusting for treatment effects - is statistically significant.

As shown in our simulation analysis, the size of the bias in exacerbation treatment effect estimates - caused by disregarding the association with treatment discontinuations - can be affected by multiple factors: the strength of the association, the size of the frailty variance (risk heterogeneity), and the treatment discontinuation rates in the different treatment arms. Moreover, the direction of the bias is determined by whether there is a higher (treatment effect is underestimated) or lower (treatment effect is overestimated) rate of discontinuations in the control/reference arm compared to the other treatment $\operatorname{arm}(\mathrm{s})$. These same characteristics were observed also in the analysis of the clinical trial datasets. The difference in exacerbation treatment effect estimates 
Table 5 Results from the joint frailty model, including selected covariates, when applied to the pooled datasets

\begin{tabular}{|c|c|c|c|c|}
\hline & \multicolumn{2}{|c|}{ Exacerbations (moderate/severe) } & \multicolumn{2}{|c|}{ Early treatment discontinuations } \\
\hline & $\begin{array}{l}\text { Pooled A-C } \\
\text { HR }(95 \% \text { Cl) }\end{array}$ & $\begin{array}{l}\text { Pooled D-E } \\
\text { HR }(95 \% \mathrm{Cl})\end{array}$ & $\begin{array}{l}\text { Pooled A-C } \\
\text { HR }(95 \% \text { Cl) }\end{array}$ & $\begin{array}{l}\text { Pooled D-E } \\
\text { HR (95\% Cl) }\end{array}$ \\
\hline \multicolumn{5}{|l|}{ Demographics } \\
\hline Age (+ 1 year $)$ & $1.00(0.99-1.00)$ & $1.00(0.99-1.00)$ & $1.01(1.00-1.02)$ & $1.01(1.00-1.03)$ \\
\hline Sex (male vs female) & & & $1.29(1.06-1.56)$ & $1.24(1.00-1.55)$ \\
\hline \multicolumn{5}{|l|}{ Region } \\
\hline Western Europe vs US & $1.10(0.91-1.34)$ & $1.56(1.28-1.90)$ & $0.59(0.43-0.82)$ & $1.27(0.86-1.87)$ \\
\hline Eastern Europe vs US & $0.49(0.42-0.57)$ & $0.61(0.52-0.71)$ & $0.21(0.16-0.28)$ & $0.45(0.34-0.61)$ \\
\hline Rest of the World vs US & $0.83(0.70-0.99)$ & $0.83(0.73-0.95)$ & $0.30(0.22-0.41)$ & $0.50(0.39-0.64)$ \\
\hline \multicolumn{5}{|l|}{ Treatment } \\
\hline BUD/FM 320 vs FM & $0.71(0.61-0.82)$ & & $0.73(0.56-0.94)$ & \\
\hline BUD/FM 160 vs FM & $0.71(0.61-0.83)$ & & $0.75(0.59-0.97)$ & \\
\hline BUD $320+F M$ vs FM & $0.57(0.42-0.77)$ & & $0.58(0.36-0.94)$ & \\
\hline BUD 320 vs FM & $0.77(0.58-1.04)$ & & $1.03(0.65-1.64)$ & \\
\hline PBO vs FM & $1.20(1.00-1.44)$ & & $1.12(0.84-1.50)$ & \\
\hline
\end{tabular}

$\mathrm{SOC}+$ RFL VS SOC

$0.88(0.80-0.97)$

$1.61(1.34-1.94)$

\section{Disease related baseline factors}

\begin{tabular}{|c|c|c|}
\hline $\begin{array}{l}\text { Exacerbation history } \\
(+1 \text { previous year) }\end{array}$ & $1.16(1.12-1.21)$ & $1.21(1.14-1.28)$ \\
\hline $\begin{array}{l}\text { Smoking history } \\
\text { (+ } 10 \text { pack-years) }\end{array}$ & $1.02(1.01-1.04)$ & \\
\hline ICS history (yes vs no) & $1.19(1.06-1.34)$ & \\
\hline $\begin{array}{l}\text { Bronchodilator history } \\
\text { (yes vs no) }\end{array}$ & $1.30(1.16-1.46)$ & \\
\hline $\mathrm{FEV}_{1}(+100 \mathrm{~mL})$ & $0.92(0.90-0.93)$ & $0.52(0.45-0.61)$ \\
\hline SGRQ total score (+ 10 points) & $1.04(1.00-1.09)$ & \\
\hline Breathlessness (+ 1 point) & $1.11(1.02-1.21)$ & \\
\hline CAT total score (+ 5 points) & & $1.09(1.05-1.13)$ \\
\hline $\begin{array}{l}\text { Use of rescue medication } \\
\text { (+ } 1 \text { puff) }\end{array}$ & $1.04(1.03-1.06)$ & $1.03(1.02-1$. \\
\hline \multicolumn{3}{|l|}{ Seasonality } \\
\hline Spring vs Autumn & $0.76(0.68-0.85)$ & $1.00(0.90-1$. \\
\hline Summer vs Autumn & $0.69(0.61-0.77)$ & $0.78(0.70-0$ \\
\hline Winter vs Autumn & $1.07(0.97-1.19)$ & $1.17(1.06-1.2)$ \\
\hline
\end{tabular}

\section{Trial effect}

$\begin{array}{ll}\text { A vs C } & 0.76(0.65-0.89) \\ \text { B vs C } & 0.85(0.70-1.03)\end{array}$

$0.86(0.67-1.10)$

$0.78(0.57-1.07)$

Evs D

$1.08(0.94-1.25)$

$1.64(1.20-2.24)$

\section{Association parameters* \\ Pooled A-C}

$1.14(\mathrm{SE}=0.07)$
$1.54(\mathrm{SE}=0.15)$
$0.7(0.53-0.93)$

$1.01(1.00-1.02)$

$1.04(1.01-1.06)$

$1.02(1.00-1.03)$

Frailty variance

Association (a)

\section{Pooled D-E}

$0.67(\mathrm{SE}=0.05)$

$0.95(\mathrm{SE}=0.17)$

BUD budesonide, FM formoterol, RFL roflumilast, ICS inhaled corticosteroids, HR hazard ratio, Cl confidence interval, SE standard error, US United States, * - all parameters significantly different from zero with $p$-value $<0.0001$ 
between the joint frailty and standard models was largest in Datasets C-E, which were the datasets showing the biggest separation between treatment arms in terms of treatment discontinuations. The absolute difference in treatment effect estimates in these datasets were between 4 and 15 percentage points, which can be regarded as clinically relevant.

In our covariate search, we identified prognostic factors of exacerbation risk that all have been previously established [31-35]. However, their impact has - to our knowledge - never been quantified using a joint frailty model, and our analysis further supports their value in predicting exacerbation risk. The use of the joint frailty model also allowed us to include the time-varying effect of seasonality in our analysis, confirming a higher risk of exacerbations during the winter months and a lower risk during summer [28].

Interestingly, several of the covariates associated to exacerbation risk were also associated to treatment discontinuation risk, supporting our finding that the two risks are associated. Including prognostic factors in the model, however, resulted in a relatively small reduction of the frailty variance, which is in line with the general understanding that known risk factors explain only a small part of COPD patient heterogeneity [34, 36]. It also highlights the importance of considering heterogeneity in the statistical analysis, even after adjusting for known covariates.

Our analysis is limited by the fact that we, according to data re-use rules and consent reasons, did not have access to data from all patients included in the original trials. Of particular note is that treatment discontinuation rates were higher in the original trials compared to the rates in our analysis datasets (see Table 2). As shown in our simulation study, an increased rate of treatment discontinuations can contribute to an increased bias in exacerbation treatment effect estimates of models disregarding the association between exacerbations and treatment discontinuations. Therefore, differences in treatment effect estimates between the joint frailty model and the shared frailty and negative binomial models would likely have been even more pronounced had we been able to use the original trial datasets.

While our analysis clearly demonstrates an overall association between the risks of exacerbation and treatment discontinuation, it is also limited by the fact that we have treated all discontinuations the same regardless of reason (e.g. adverse events, disease worsening, or lost to followup). Further work may be done to exclude discontinuations known to be unrelated to the disease or trial (although such a classification can sometimes be difficult or not possible due to missing information). Particularly, this may impact the analysis of the roflumilast data, where GI adverse events lead to discontinuations - predominantly during the first month of treatment - and where the association to exacerbation risk can be questioned. Indeed, the overall risk association was estimated to be weaker in Datasets D-E. Further work could also include exploring alternative model structures to describe the association between exacerbation and discontinuation risks.

\section{Conclusions}

We have identified a significant association between the risks of exacerbation and early treatment discontinuation in COPD clinical trials and show that treatment discontinuations per se contribute important information on exacerbation outcomes - results we believe add significant value to the discussion on missing data in COPD and clinical trials in general $[6,10]$. We regard the joint frailty model as a useful approach to study the impact of discontinuations on exacerbation treatment effect estimates and recommend it to be used as a complement to standard analyses in COPD clinical trials, e.g. when differentiating the clinical benefit of an effective and less effective treatment (assay sensitivity) [37].

\section{Supplementary information}

Supplementary information accompanies this paper at https://doi.org/10. 1186/s12931-020-01419-8.

Additional file 1. Additional details on datasets, statistical methods, simulation study, and additional results from the clinical trial data

\section{Abbreviations \\ AG: Andersen-Gill, bid: twice daily; BUD: Budesonide; CAT: Chronic

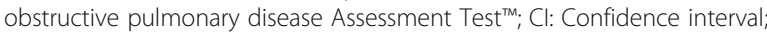 COPD: Chronic obstructive pulmonary disease; DPI: Dry powder inhaler; $\mathrm{FEV}_{1}$ : Forced expiratory volume in $1 \mathrm{~s}$; FM: Formoterol; Gl: Gastrointestinal; HR: Hazard ratio; ICS: Inhaled corticosteroid; LABA: Long-acting beta agonist; LAMA: Long-acting muscarinic receptor antagonist; MAR: Missing at random; PBO: Placebo; pMDI: Pressurized metered-dose inhaler; PROs: Patient reported outcomes; RFL: Roflumilast; SABA: Short-acting beta agonists; SE: Standard error; SGRQ: St. George's Questionnaire; SOC: Standard of care; US: United States}

\section{Acknowledgements}

AK is a fellow of the AstraZeneca postdoc programme. The authors wish to thank Ulrika Wählby Hamrén (AstraZeneca) for providing valuable comments on the manuscript.

\section{Authors' contributions \\ $A K, R P, S R$, UGE and AJ contributed to the conception of the work. AK, RP, $V R$ and $A J$ contributed to the design of the work. Analyses were done by AK, $\mathrm{RP}$ and $\mathrm{AJ}$. All authors contributed to the interpretation of data. AK, RP and AJ wrote the manuscript. All authors revised it critically for important intellectual content and gave their final approval.}

\section{Funding}

The study was funded by AstraZeneca. AstraZeneca reviewed the publication, without influencing the opinions of the authors, to ensure medical and scientific accuracy, and the protection of intellectual property.

\section{Availability of data and materials}

De-identified patient data underlying the findings described in this manuscript can be requested in accordance with AstraZeneca's data sharing policy described at https://astrazenecagrouptrials.pharmacm.com/ST/ Submission/Disclosure. The $\mathrm{R}$ code that supports the findings of this analysis is available on request from the corresponding author. 


\section{Ethics approval and consent to participate}

This was a post hoc analysis of five clinical trials; the original study protocols were approved by local institutional review boards and ethics committees, and conducted in accordance with the Declaration of Helsinki, International Conference on Harmonization/Good Clinical Practice, and applicable local regulations. All patients provided their consent.

\section{Consent for publication}

Not applicable.

\section{Competing interests}

At the time this work was conducted AK, RP, SR, UGE, and AJ were employees of AstraZeneca. VR has no conflicts of interest to declare.

\section{Author details}

'Clinical Pharmacology and Safety Sciences, BioPharmaceuticals R\&D, AstraZeneca, Gothenburg, Sweden. 'Biostatistics Team, INSERM CR1219, University of Bordeaux, Bordeaux, France. ${ }^{3}$ BioPharmaceuticals R\&D, AstraZeneca, Cambridge, UK. ${ }^{4}$ University of Nebraska Medical Center, Omaha, NE, USA. ${ }^{5}$ BioPharma Early Biometrics and Statistical Innovation, Data Science \& Al, BioPharmaceuticals R\&D, AstraZeneca, Pepparedsleden 1, SE-431 83 Mölndal, Sweden.

Received: 17 October 2019 Accepted: 9 June 2020

Published online: 22 June 2020

\section{References}

1. Decramer M, Molenberghs G, Liu D, Celli B, Kesten S, Lystig T, et al. Premature discontinuation during the UPLIFT study. Respir Med. 2011;105(10):1523-30.

2. Eriksson G, Calverley PM, Jenkins CR, Anzueto AR, Make BJ, Lindberg M, et al. The effect of COPD severity and study duration on exacerbation outcome in randomized controlled trials. Int J Chron Obstruct Pulmon Dis. 2017;12:1457-68.

3. Suissa S, Ernst P, Vandemheen KL, Aaron SD. Methodological issues in therapeutic trials of COPD. Eur Respir J. 2008;31(5):927-33.

4. Bell ML, Kenward MG, Fairclough DL, Horton NJ. Differential dropout and bias in randomised controlled trials: when it matters and when it may not. BMJ. 2013;346:e8668.

5. Kesten S, Plautz M, Piquette CA, Habib MP, Niewoehner DE. Premature discontinuation of patients: a potential bias in COPD clinical trials. Eur Respir J. 2007;30(5):898-906.

6. Little RJ, D'Agostino R, Cohen ML, Dickersin K, Emerson SS, Farrar JT, et al. The prevention and treatment of missing data in clinical trials. N Engl J Med. 2012;367(14):1355-60.

7. Keene ON, Calverley PM, Jones PW, Vestbo J, Anderson JA. Statistical analysis of exacerbation rates in COPD: TRISTAN and ISOLDE revisited. Eur Respir J. 2008;32(1):17-24.

8. Aaron SD, Fergusson D, Marks GB, Suissa S, Vandemheen KL, Doucette S, et al. Counting, analysing and reporting exacerbations of COPD in randomised controlled trials. Thorax. 2008;63(2):122-8.

9. Vestbo J. Counting, analysing and reporting exacerbations of COPD in randomised controlled trials. Thorax. 2008;63(8):749-50 author reply 50.

10. Keene ON, Roger JH, Hartley BF, Kenward MG. Missing data sensitivity analysis for recurrent event data using controlled imputation. Pharm Stat. 2014;13(4):258-64.

11. Liu D, Menjoge S. Statistical analysis of chronic obstructive pulmonary disease (COPD) exacerbations. Eur Respir J. 2008;32(5):1422-3 author reply 3.

12. Watz H, Tetzlaff K, Wouters EF, Kirsten A, Magnussen H, Rodriguez-Roisin R, et al. Blood eosinophil count and exacerbations in severe chronic obstructive pulmonary disease after withdrawal of inhaled corticosteroids: a post-hoc analysis of the WISDOM trial. Lancet Respir Med. 2016;4(5):390-8.

13. Martinez FJ, Calverley PM, Goehring UM, Brose M, Fabbri LM, Rabe KF. Effect of roflumilast on exacerbations in patients with severe chronic obstructive pulmonary disease uncontrolled by combination therapy (REACT): a multicentre randomised controlled trial. Lancet. 2015;385(9971):857-66.

14. Martinez FJ, Rabe KF, Sethi S, Pizzichini E, Mclvor A, Anzueto A, et al. Effect of Roflumilast and inhaled corticosteroid/long-acting beta2-agonist on chronic obstructive pulmonary disease exacerbations (RE(2) SPOND). A randomized clinical trial. Am J Respir Crit Care Med. 2016;194(5):559-67.

15. Cook RJ, Lawless JF. The statistical analysis of recurrent events. New York. London: Springer; 2011.
16. Andersen PK, Gill RD. Cox's regression model for counting processes: a large sample study. Ann Stat. 1982;10(4):1100-20.

17. International Council for Harmonisation of Technical Requirements for Pharmaceuticals for Human Use. Addendum on estimands and sensitivity analysis in clinical trials to the guideline on statistical principles for clinical trials E9 (R1). 20 November 2019. https://database.ich.org/sites/default/files/ E9-R1_Step4_Guideline_2019_1203.pdf.

18. Keene ON, Ruberg S, Schacht A, Akacha M, Lawrance R, Berglind A, et al. What matters most? Different stakeholder perspectives on estimands for an invented case study in COPD. Pharm Stat. 2020.

19. Roger JH, Bratton DJ, Mayer B, Abellan JJ, Keene ON. Treatment policy estimands for recurrent event data using data collected after cessation of randomised treatment. Pharm Stat. 2019;18(1):85-95.

20. Liu L, Wolfe RA, Huang $X$. Shared frailty models for recurrent events and a terminal event. Biometrics. 2004;60(3):747-56.

21. Rondeau V, Mathoulin-Pelissier S, Jacqmin-Gadda H, Brouste V, Soubeyran P. Joint frailty models for recurring events and death using maximum penalized likelihood estimation: application on cancer events. Biostatistics. 2007;8(4):708-21.

22. Rennard SI, Tashkin DP, McElhattan J, Goldman M, Ramachandran S, Martin UJ, et al. Efficacy and tolerability of budesonide/formoterol in one hydrofluoroalkane pressurized metered-dose inhaler in patients with chronic obstructive pulmonary disease: results from a 1-year randomized controlled clinical trial. Drugs. 2009;69(5):549-65.

23. Sharafkhaneh A, Southard JG, Goldman M, Uryniak T, Martin UJ. Effect of budesonide/formoterol pMDI on COPD exacerbations: a double-blind, randomized study. Respir Med. 2012;106(2):257-68.

24. Tashkin DP, Rennard SI, Martin P, Ramachandran S, Martin UJ, Silkoff PE, et al. Efficacy and safety of budesonide and formoterol in one pressurized metered-dose inhaler in patients with moderate to very severe chronic obstructive pulmonary disease: results of a 6-month randomized clinical trial. Drugs. 2008;68(14):1975-2000.

25. R Development Core team. R: a language and environment for statistical computing. R Foundation for Statistical Computing; 2017. http://www.r-project.org.

26. Król A, Mauguen A, Mazroui Y, Laurent A, Michiels S, Rondeau V. Tutorial in Joint Modeling and Prediction: A Statistical Software for Correlated Longitudinal Outcomes, Recurrent Events and a Terminal Event. J Stat Software. 2017;1:3.

27. Tibshirani R. The lasso method for variable selection in the cox model. Stat Med. 1997;16(4):385-95.

28. Jenkins CR, Celli B, Anderson JA, Ferguson GT, Jones PW, Vestbo J, et al. Seasonality and determinants of moderate and severe COPD exacerbations in the TORCH study. Eur Respir J. 2012;39(1):38-45.

29. Calverley PM, Spencer S, Willits L, Burge PS, Jones PW, Group IS. Withdrawal from treatment as an outcome in the ISOLDE study of COPD. Chest. 2003;124(4):1350-6.

30. Rogliani P, Calzetta L, Cazzola M, Matera MG. Drug safety evaluation of roflumilast for the treatment of COPD: a meta-analysis. Expert Opin Drug Saf. 2016;15(8):1133-46.

31. Make BJ, Eriksson G, Calverley PM, Jenkins CR, Postma DS, Peterson S, et al. A score to predict short-term risk of COPD exacerbations (SCOPEX). Int J Chron Obstruct Pulmon Dis. 2015;10:201-9.

32. Jenkins CR, Postma DS, Anzueto AR, Make BJ, Peterson S, Eriksson G, et al. Reliever salbutamol use as a measure of exacerbation risk in chronic obstructive pulmonary disease. BMC Pulm Med. 2015;15:97.

33. Calverley PM, Tetzlaff K, Dusser D, Wise RA, Mueller A, Metzdorf N, et al. Determinants of exacerbation risk in patients with COPD in the TIOSPIR study. Int J Chron Obstruct Pulmon Dis. 2017;12:3391-405.

34. Hurst JR, Vestbo J, Anzueto A, Locantore N, Mullerova H, Tal-Singer R, et al. Susceptibility to exacerbation in chronic obstructive pulmonary disease. N Engl J Med. 2010;363(12):1128-38.

35. Lee SD, Huang MS, Kang J, Lin CH, Park MJ, Oh YM, et al. The COPD assessment test (CAT) assists prediction of COPD exacerbations in high-risk patients. Respir Med. 2014;108(4):600-8.

36. Burgel PR, Paillasseur JL, Caillaud D, Tillie-Leblond I, Chanez P, Escamilla R, et al. Clinical COPD phenotypes: a novel approach using principal component and cluster analyses. Eur Respir J. 2010;36(3):531-9.

37. European Medicines Agency. Guideline on clinical investigation of medicinal products in the treatment of chronic obstructive pulmonary disease (COPD). https://www.ema.europa.eu; 2012. https://www.ema.europa.eu.

\section{Publisher's Note}

Springer Nature remains neutral with regard to jurisdictional claims in published maps and institutional affiliations. 